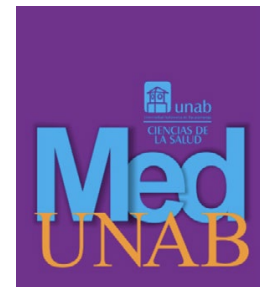

REVISTA DE LA FACULTAD

DE CIENCIAS DE LA SALUD

Vol. 24(3): 387-391, diciembre 2021 - marzo 2022

i-SSN 0123-7047

e-ISSN 2382-4603

\title{
Actualización de la distribución geográfica de triatominos en el departamento del Putumayo
}

Update to the geographical distribution of triatomines in the Department of Putumayo Atualização da distribuição geográfica de triatomíneos no Departamento de Putumayo

\section{Keila Ortiz-Canamejoy, Biól., Esp. ${ }^{1}$ (D)}

1. Bióloga, Especialista en Epidemiología, Responsable Técnica del área de Entomología. Gobernación del Putumayo, Secretaría de Salud Departamental, Laboratorio de Salud Pública, área Entomología. Mocoa, Putumayo, Colombia.

Correspondencia. Keila Ortiz Canamejoy. Barrio Progreso Calle 11A No. 11- 11 Mocoa, Putumayo 8600001. Email. keilaortiz70@gmail.com

\section{INFORMACIÓN DEL ARTÍCULO:}

Artículo recibido: 08 de marzo de 2021

Artículo aceptado: 05 de octubre de 2021

DOI: https://doi.org/10.29375/01237047.4103

Cómo citar: Ortiz-Canamejoy K. Actualización de la distribución geográfica de triatominos en el departamento del Putumayo. MedUNAB [Internet]. 2021;24(3): 387-391. doi: https://doi.org/10.29375/01237047.4103

Palabras claves:

Triatominae; Enfermedad de Chagas; Infección por Trypanosoma cruzi; Infecciones; Insectos Vectores; Colombia.

Keywords:

Triatominae; Chagas Disease; Trypanosoma cruzi; Infections; Insect Vectors;

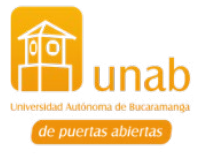

Colombia.

Palavras-chave:

Triatominae; Doença de Chagas; Infecção por Trypanosoma cruzi; Infecções; Insetos Vetores; Colômbia. 
La enfermedad de Chagas es una zoonosis producida por la infección del Trypanosoma cruzi (T. cruzi) (1), cuya principal vía de transmisión es vectorial (2). Esta enfermedad se caracteriza por ser una infección crónica que puede ocasionar daños cardiacos, digestivos y neurológicos irreversibles (3).

En el departamento del Putumayo, de acuerdo con los datos del Sistema de Vigilancia Epidemiológica (SIVIGILA), entre el año 2015 y el 2020, se han notificado 19 casos de Chagas crónico y 4 casos de Chagas agudos (4).

Por este motivo resulta de gran interés compartir con los lectores de la revista MedUNAB la actualización de la distribución geográfica de los triatominos, vectores de la enfermedad de Chagas, y establecer el riesgo epidemiológico que representan para la población Putumayense, donde hay hallazgos de gran importancia porque se identifican especies en municipios y localidades donde antes no se conocían.
Se hizo un estudio retrospectivo de las bases de datos de la Unidad de Entomología del Laboratorio de Salud Pública, de la Secretaria de Salud de Putumayo, entre los años 2016 y 2020.

La identificación taxonómica la realizó la profesional de la unidad de Entomología del Laboratorio de Salud Pública, mediante la clave de Lent \& Wygodzinsky 1979 y, la confirmación, la realizó el Grupo de Entomología del Instituto Nacional de Salud.

Durante los años 2016 a 2020, se identificaron en el laboratorio de entomología, un total de 52 triatominos que corresponden a cuatro especies: Rhodnius prolixus, Rhodnius pictipes, Panstrogylus geniculatus y Eratirus mucronatus.

Los insectos fueron colectados en 30 localidades, de 6 municipios: Puerto Asís, Orito, Villagarzón, Puerto Guzmán, Mocoa y Puerto Leguízamo (Figura 1 y Tabla 1).

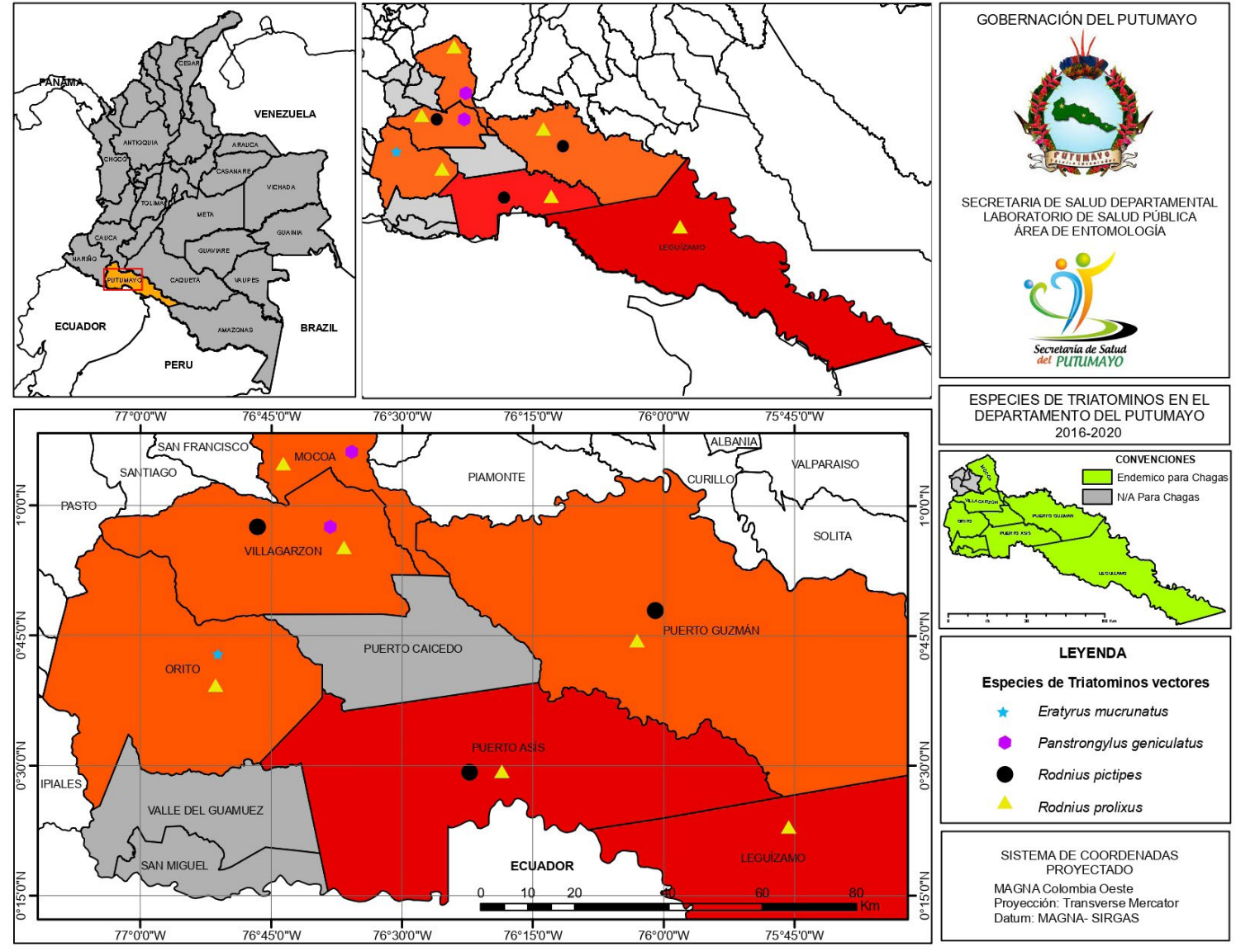

Figura 1: Mapa de ubicación de las especies de triatominos en el Putumayo.

Fuente: elaborado por la autora, Unidad de Entomología, Laboratorio de Salud Pública de Putumayo, Sistemas de Información Geográfica del Putumayo - 2020 
Las especies $R$. pictipes y $P$. geniculatus son nuevos registros para el municipio de Villagarzón, y Rhodnius prolixus para el municipio de Puerto Leguízamo, por lo que se amplía la distribución geográfica de las especies de triatominos en el departamento del Putumayo.

Del total de ejemplares colectados, el $75 \%$ correspondieron a capturas en el interior de las viviendas, el $15 \%$ en el peridomicilio y el $10 \%$ de los registros no reportan el sitio de captura.
La transmisión vectorial de la enfermedad de Chagas, ha estado asociada a los ambientes rurales. Sin embargo, en este estudio se puede apreciar la presencia de triatominos vectores en barrios de los diferentes municipios (Tabla 1). El comportamiento dispersivo de los triatominos se ve favorecido por situaciones como la electrificación, la construcción de carreteras y por el desarrollo urbanístico en zonas rurales, lo cual desplaza no sólo los reservorios, que son su fuente de alimento, sino también a los insectos mismos, quienes, por su mal estado nutricional, vuelan en busca de otras alternativas como los animales domésticos y el hombre (5).

Tabla 1. Distribución geográfica de las especies de triatominos presentes en el departamento del Putumayo y su infección natural con T. cruzi entre el 2016 y el 2020.

\begin{tabular}{|c|c|c|c|c|c|}
\hline Municipios & Especie & Localidades & Latitud & Longitud & Altura \\
\hline \multirow{13}{*}{ Puerto Asís } & \multirow{9}{*}{ Rhodnius prolixus } & Barrio Corralito de piedra & 0.500608 & -76.496184 & 255 \\
\hline & & Barrio Los Cristales & 0.500916 & -76.496168 & 255 \\
\hline & & Barrio Altos del Cedral & 0.49333 & -76.493278 & 255 \\
\hline & & Barrio Colinas & 0.494971 & -76.492072 & 256 \\
\hline & & Vereda Montañita & 0.268756 & -76.460246 & 299 \\
\hline & & Vereda Alea Sevilla & 0.460223 & -76.299705 & 260 \\
\hline & & Vereda La Pradera & 0.380975 & -76.553056 & 271 \\
\hline & & Vereda Agualongo* & 0.400381 & -76.376755 & 260 \\
\hline & & Vereda Brisas de San Vicente & $\begin{array}{c}\text { SIN } \\
\text { DATOS }\end{array}$ & $\begin{array}{c}\text { SIN } \\
\text { DATOS }\end{array}$ & $\begin{array}{c}\text { SIN } \\
\text { DATOS }\end{array}$ \\
\hline & \multirow{4}{*}{ Rhodnius pictipes } & Barrio Altos del Cedral & 0.49333 & -76.493278 & 255 \\
\hline & & Barrio centro & 0.495056 & -76.498516 & 256 \\
\hline & & Barrio Colinas* & 0.494971 & -76.492072 & 256 \\
\hline & & Vereda Agualongo* & 0.400381 & -76.376755 & 260 \\
\hline \multirow{2}{*}{ Orito } & Rhodnius prolixus & Resguardo awa la Turbia & 0.784325 & -76.841781 & 474 \\
\hline & Eratirus mucronatus & Resguardo awa la Turbia & 0.784325 & -76.841781 & 474 \\
\hline \multirow{5}{*}{ Villagarzón } & \multirow{2}{*}{ Rhodnius prolixus } & Vereda Alto Mecaya & 0.965024 & -76.583100 & 360 \\
\hline & & Vereda Brisas de San Vicente & 0.912342 & -76.665488 & 420 \\
\hline & \multirow{2}{*}{ Rhodnius pictipes } & Vereda Brisas de San Vicente & 0.912342 & -76.665488 & 420 \\
\hline & & Vereda Alto Mecaya & 0.965024 & -76.583100 & 360 \\
\hline & Panstrogylus geniculatus & Vereda Alto Mecaya & 0.965024 & -76.583100 & 360 \\
\hline
\end{tabular}




\begin{tabular}{|c|c|c|c|c|c|}
\hline \multirow{6}{*}{ Puerto Guzmán } & \multirow{4}{*}{ Rhodnius prolixus } & $\begin{array}{l}\text { Barrio El Poblado } \\
\text { (dos individuos) } 1^{*}\end{array}$ & 0.958533 & -76.411 .928 & 275 \\
\hline & & Resguardo Aguaditas & 0.957531 & -76.401737 & 272 \\
\hline & & $\begin{array}{l}\text { Barrio Prados (tres } \\
\text { individuos) } 1^{*}\end{array}$ & 0.959066 & -76.404039 & 273 \\
\hline & & Vereda La Torre & 0.81736 & -76.117491 & 265 \\
\hline & \multirow{2}{*}{ Rhodnius pictipes } & Barrio Edisón Mora* & 0.966629 & -76.404045 & 274 \\
\hline & & Casas Cabildo & $\begin{array}{c}\text { SIN } \\
\text { DATOS }\end{array}$ & $\begin{array}{c}\text { SIN } \\
\text { DATOS }\end{array}$ & $\begin{array}{c}\text { SIN } \\
\text { DATOS }\end{array}$ \\
\hline \multirow{3}{*}{ Mocoa } & \multirow{2}{*}{ Rhodnius prolixus } & Barrio Villa Diana & 1.157672 & -76.646911 & 608 \\
\hline & & Vereda El Pepino & 1.082608 & -76.656848 & 674 \\
\hline & $\begin{array}{l}\text { Panstrongylus } \\
\text { geniculatus }\end{array}$ & Barrio Álamos & 1.150761 & -76.644325 & 580 \\
\hline Puerto Leguízamo & Rhodnius prolixus & Vereda Puerto Nariño* & 0.339278 & -74.631064 & 186 \\
\hline
\end{tabular}

Debido al comportamiento intradomiciliario de los triatominos vectores, en barrios en Putumayo, se pone de manifiesto que esta enfermedad no es exclusiva de la pobreza, como se menciona en diferentes estudios (69). En la mayoría de las viviendas de los barrios donde se encontraron los triatominos, incluso infectados con T. cruzi, las viviendas no presentan características, ni materiales de riesgo asociado con la infestación de triatominos, la ubicación de las viviendas de estos barrios es muy cercana a bosques secundarios, lo que facilita el ingreso de los triatominos a las viviendas.

En este estudio $\boldsymbol{R}$. prolixus fue una de las especies encontrada con infección natural de T. cruzi, la de mayor densidad, y el $79 \%$ de los especímenes colectados, fueron hallados en el intradomicilio, en una amplia distribución de 19 localidades en los municipios de Puerto Asís, Orito, Villagarzón, Puerto Guzmán, Mocoa y Puerto Leguizamo. En consecuencia, es razonable incriminar a esta especie como la especie vector más importante en el departamento del Putumayo.

R. pictipes, es la segunda especie con mayor densidad, y presentó infección natural de T. cruzi. El 55\% de los especímenes, fueron encontrados en el intradomicilio y la especie se identificó en 8 localidades de los municipios de Puerto Asís, Villagarzón y Puerto Guzmán.
$\boldsymbol{P}$. geniculatus, es una especie principalmente extradomiciliaria que eventualmente llega a las casas atraída por la luz. Fue hallada en el intradomicilio negativa para $T$. cruzi y se ubicó en dos localidades de los municipios de Villagarzón y Mocoa.

Por último, la especie $\boldsymbol{E}$. mucronatus se identifica en el municipio de Orito (10).

Los anteriores hallazgos, encontrados en el presente estudio, permitieron establecer zonas de alto riesgo, las cuales han sido intervenidas por la Secretaría de Salud Departamental de Putumayo.

\section{Agradecimientos}

Al Dr. Gabriel Parra por la revisión al documento; al Programa de Enfermedades Transmitidas por Vectores (ETV) y Zoonosis por el apoyo en el trabajo de campo; al Grupo de Entomología de la Subdirección Red Nacional de Laboratorios del Instituto Nacional de Salud por su apoyo en la confirmación de los especímenes; al Dr. Buanerges Florencio Rosero Peña, Gobernador del Putumayo y a la Dra. Adriana Lucía Médicis, Secretaria de Salud Departamental, por la gestión y apoyo en la realización de esta Investigación. 


\section{Conflictos de intereses}

La autora declara no tener conflictos de intereses.

\section{Financiación}

Para la realización de este estudio, no existió ningún tipo de financiación externa a los autores.

\section{Referencias}

1. López-Cárdenas J, González-Bravo FE, SalazarSchettino PM. Distribución Espacial de Vectores de la Enfermedad de Chagas en el Estado de Guanajuato 1998-2000. Acta Universitaria [Internet]. 2002 [consultado 6 enero de 2021];12(3):64-69. doi: https://doi.org/10.15174/au.2002.284

2. Parra-Henao G, Angulo V, Jaramillo N, Restrepo M. Triatominos (Hemiptera: Reduviidae) de la Sierra Nevada de Santa Marta, Colombia. Aspectos epidemiológicos, entomológicos y de distribución. Rev CES Med [Internet]. 2009;23(1):17-26. Recuperado a partir de: http://www.scielo.org.co/scielo.php?script=sci arttext\&pid=S0120-87052009000100003

3. Ayala-Hoyos CJ, Hernández-Mendoza CM, EyesEscalante M, Romero-Ricardo LR, ÁlvarezRodríguez RA, Blanco-Tuirán P. Detección de infección natural por Trypanosoma cruzi (Trypanosomatidae) en triatominos del municipio de Colosó, Colombia. Acta biol. Colomb [Internet]. 2019;24(1):180-184.doi:http://dx.doi.org/10.15446/ abc.v24n1.72306

4. Secretaría de Salud del departamento del Putumayo. Área de Epidemiología. Casos reportados por software SIVIGILA, Sistema de Vigilancia
Epidemiológica durante los años 2015 a semana 53 de 2020. Recuperado a partir de: https://www. ins.gov.co/buscador-eventos/Paginas/Vista-BoletinEpidemilogico.aspx

5. Castillo D, Wolff M. Aspecto del comportamiento de los triatominos (Hemiptera: Reduviidae), vectores de la enfermedad de Chagas. Biomédica [Internet]. 2000;20(1):59-64. doi: https://doi.org/10.7705/ biomedica.v20i1.1048

6. Pinto JC. Reseña histórica de los conocimientos sobre la enfermedad de Chagas y reflexiones sobre algunos aspectos políticos y socioeconómicos de la endemia en el contexto latinoamericano. Rev Fed Argent Cardiol. 1988;17:121.

7. Rivero-Gairaud I. Enfermedad de Chagas. Enfermedades tropicales. Rev. méd. Costa Rica Centroam. [Internet]. 2016 [consultado 5 de mayo de 2021];83(619):298. Recuperado a partir de: https://www.medigraphic.com/pdfs/revmedcoscen/ rmc-2016/rmc162r.pdf

8. Flórez C, Guasmayan L, Cortés L, Caicedo A, Beltrán M, Muñoz L. Enfermedad de Chagas y su seroprevalencia en tres departamentos de la Amazonia colombiana. NOVA. 2016 [consultado 2 de febrero de 2021];14(26):41. doi: https://doi. org/10.22490/24629448.1749

9. Cazorla-Perfetti D. Revisión de los vectores de la enfermedad de Chagas en Venezuela (hemiptera - heteroptera, Reduviidae, triatominae). Saber [Internet]. 2016 [consultado 1 de marzo de 2021];28(3):395. Recuperado a partir de: http:// ve.scielo.org/pdf/saber/v28n3/art03.pdf

10. Ortiz, K. Primer registro de Eratyrus mucronatus Stal, 1859: vector de la enfermedad de Chagas en el departamento del Putumayo, Colombia. MedUNAB [Internet]. 2019 [consultado 6 de marzo de 2021];21(3):308-313. doi: https://doi. org/10.29375/01237047.3583 